\title{
Clinical Characteristics and Prognosis of COPD Patients Hospitalized with SARS-CoV-2
}

This article was published in the following Dove Press journal:

International Journal of Chronic Obstructive Pulmonary Disease

\author{
María Gómez Antúnez' \\ Antonio Muiño Míguez' \\ Alejandro David Bendala Estrada' \\ Guillermo Maestro de la Calle (iD ${ }^{2}$ \\ Daniel Monge Monge ${ }^{3}$ \\ Ramón Boixeda (iD) ${ }^{4}$ \\ Javier Ena (D) ${ }^{5}$ \\ Carmen Mella Pérez ${ }^{6}$ \\ Juan Miguel Anton Santos ${ }^{7}{ }^{7}$ \\ Carlos Lumbreras Bermejo ${ }^{2}$ \\ On behalf of the SEMI-COVID-19 \\ Network \\ 'Internal Medicine Department, Gregorio \\ Marañón University Hospital, Madrid, \\ Spain; ${ }^{2}$ Internal Medicine Department, 12 \\ de Octubre University Hospital, Madrid, \\ Spain; ${ }^{3}$ Internal Medicine Department, \\ Segovia Hospital Complex, Segovia, \\ Spain; ${ }^{4}$ Internal Medicine Department, \\ Mataró Hospital, Mataró, Barcelona, \\ Spain; ${ }^{5}$ Internal Medicine Department, \\ Marina Baixa Hospital, Villajoyosa, \\ Alicante, Spain; ${ }^{6}$ Internal Medicine \\ Department, Ferrol University Hospita \\ Complex, Ferrol, A Coruña, Spain; \\ ${ }^{7}$ Internal Medicine Department, Infanta \\ Cristina University Hospital, Parla, \\ Madrid, Spain
}

Objective: To describe the characteristics and prognosis of patients with COPD admitted to the hospital due to SARS-CoV-2 infection.

Methods: The SEMI-COVID registry is an ongoing retrospective cohort comprising consecutive COVID-19 patients hospitalized in Spain since the beginning of the pandemic in March 2020. Data on demographics, clinical characteristics, comorbidities, laboratory tests, radiology, treatment, and progress are collected. Patients with COPD were selected and compared to patients without COPD. Factors associated with a poor prognosis were analyzed. Results: Of the 10,420 patients included in the SEMI-COVID registry as of May 21, 2020, $746(7.16 \%)$ had a diagnosis of COPD. Patients with COPD are older than those without COPD (77 years vs 68 years) and more frequently male. They have more comorbidities (hypertension, hyperlipidemia, diabetes mellitus, atrial fibrillation, heart failure, ischemic heart disease, peripheral vascular disease, kidney failure) and a higher Charlson Comorbidity Index ( 2 vs $1, p<0.001$ ). The mortality rate in COPD patients was $38.3 \%$ compared to $19.2 \%$ in patients without COPD $(\mathrm{p}<0.001)$. Male sex, a history of hypertension, heart failure, moderate-severe chronic kidney disease, presence of cerebrovascular disease with sequelae, degenerative neurological disease, dementia, functional dependence, and a higher Charlson Comorbidity Index have been associated with increased mortality due to COVID-19 in COPD patients. Survival was higher among patients with COPD who were treated with hydroxychloroquine $(87.1 \%$ vs $74.9 \%, \mathrm{p}<0.001)$ and with macrolides $(57.9 \%$ vs $50 \%$, $\mathrm{p}<0.037$ ). Neither prone positioning nor non-invasive mechanical ventilation, high-flow nasal cannula, or invasive mechanical ventilation were associated with a better prognosis. Conclusion: COPD patients admitted to the hospital with SARS-CoV-2 infection have more severe disease and a worse prognosis than non-COPD patients.

Keywords: SARS-CoV-2, coronavirus, COVID-19, COPD

\section{Introduction}

In December 2019, cases of pneumonia caused by a new strain of coronavirus, severe acute respiratory syndrome coronavirus 2 (SARS-CoV-2), were described in Wuhan, China. This was the origin of the COVID-19 pandemic declared by the World Health Organization (WHO).

Chronic obstructive pulmonary disease (COPD) is defined as the presence of persistent respiratory symptoms and limitation of air flow due to abnormalities of the airway and/or alveolar airways. It is often caused by significant exposure to harmful particles and gases. It is known that these patients have high comorbidity and that a hospital admission implies a deterioration in their quality of life and greater morbidity and mortality. ${ }^{1,2}$
Correspondence: María Gómez Antúnez Internal Medicine Department, Gregorio Marañón University Hospital, Doctor Esquerdo 46, Madrid 28007, Spain Tel +34 654765704

Email mgantunez@salud.madrid.org
International Journal of Chronic Obstructive Pulmonary Disease 2020:I5 3433-3445

3433

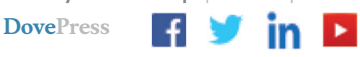


Comorbidities are considered risk factors for SARS$\mathrm{CoV}-2$ infection and are associated with greater severity of the disease and a worse prognosis. Patients with COPD have been classified as an at-risk population. ${ }^{3,4}$

SARS-CoV-2 spike (S) protein binds angiotensin converting enzyme 2 (ACE2) and together with host transmembrane serine protease 2 (TMPRSS2) promotes cell entry. The expression of ACE2 and TMPRSS 2 has been identified in lung type II pneumocytes, ileal absorptive enterocytes, and nasal goblet secretory cells. Increased expression of genes related to ACE2 and CD147 has been described in asthma, COPD, hypertension, smoking, obesity, and male gender status. This may predispose individuals to an increased risk of coronavirus respiratory tract infections in active smokers and virus-related exacerbations in patients with COPD. ${ }^{5-7}$

In mice, ACE2 and angiotensin II receptor type 2 (AT2) have been shown to protect them from severe acute lung injury induced by acid aspiration or sepsis, whereas ACE, angiotensin II and the angiotensin II type 1a receptor (AT1a) induces lung oedema and impair lung function. The same authors show that ACE-deficient mice show milder disease and that recombinant ACE2 can protect them from severe acute lung injury. ${ }^{8}$

The altered expression of these receptors could contribute to the patterns of morbidity and severity of COVID-19.

Two reviews have been published on this issue. In the first review, which is one of 11 general case series that aim to assess the prevalence of severe COVID-19 in patients with COPD who are smokers, concluded that COPD and a history of active smoking implied a worse prognosis. ${ }^{9}$ In the second review regarding COPD patients with COVID19 , the risk of severe disease (63\%) and mortality $(60 \%)$ was high, indicating that these patients have an increased risk of serious complications and death. ${ }^{10}$

In order to better manage patients with COPD who require hospitalization due to SARS-CoV-2 infection, we must know the predisposing factors, observe the clinical presentation, provide guidelines on appropriate treatment, and monitor their progress and prognosis in order to provide them with the best therapeutic management.

In Spain, at least half of COPD patients are hospitalized in internal medicine departments. Furthermore, the majority of patients hospitalized for SARS-CoV-2 are cared for in internal medicine units. This makes the collection of information from these departments feasible. ${ }^{11}$
The aim of this work is to analyze the characteristics and prognosis of COPD patients admitted to the hospital due to SARS-CoV-2 infection.

\section{Materials and Methods Observational Study}

The SEMI-COVID-19 registry is an ongoing retrospective cohort comprising consecutive patients hospitalized in 150 hospitals in Spain from March 2020 with confirmed COVID-19 disease who died during hospitalization or were discharged. ${ }^{12}$ Inclusion began on March 27 and is still ongoing. A complete list of the SEMI-COVID-19 Network members is provided in the Appendix.

All consecutive patients with confirmed SARS-CoV-2 infection who were discharged after hospitalization or who died were eligible for inclusion in the registry. COVID-19 disease was confirmed either by a positive result on realtime polymerase chain reaction (RT-PCR) testing of a nasopharyngeal or sputum sample or by a positive result on serological testing and a clinically compatible presentation. Inclusion criteria for the registry were: a) patient age $\geq 18$ years, b) a confirmed diagnosis of COVID-19, c) first hospital admission in a Spanish hospital participating in the registry, d) hospital discharge or in-hospital death.

Patients were treated at their attending physician's discretion, according to local protocols and clinical judgment.

The data is retrospectively collected from medical records by clinical researchers from across the country. Data are collected on almost 300 variables, grouped into several sections: (1) inclusion criteria; (2) epidemiological data; (3) RT-PCR and serology; (4) personal history and previous medication; (5) symptoms and physical examination upon admission; (6) laboratory tests (arterial blood gas, biochemical analysis, complete blood count, coagulation) and imaging on admission; (7) additional data seven days after admission or admission to an intensive care unit; (8) pharmacological treatment during admission (antivirals, immunomodulators, antibiotics) and ventilatory support; (9) complications during hospitalization, and (10) evolution after discharge and/or 30 days after diagnosis. The variables in the registry have been described previously. ${ }^{12}$

An online electronic data capture system (DCS) has been developed. The DCS includes a database administrator and a set of procedures for data verification. Patients are de-identified in the registry, identifiable data are dissociated and pseudonymised using an alphanumeric code. 
Each investigator maintains a protected patient registry in order to verify data and control quality. The database platform is hosted on a secure server, both the database and each client-server transfer being encrypted. The pseudonymisation system allows patient privacy to be respected while complying with ethical considerations and data protection regulations.

The Spanish Society of Internal Medicine (SEMI) is the promoter of this study. The investigators who coordinate the study in each hospital are partners of SEMI and agreed to participate in the study voluntarily and without any remuneration. The monitoring of the study is carried out by the scientific committee of the same and an independent agency. Logistical coordination and data analysis are also carried out by independent agencies.

For this work, the patients included in the SEMICOVID-19 Registry who had the diagnosis of COPD recorded in their medical history as a personal history were selected, without the obligation to have a diagnostic spirometry. In those who had a spirometry reflected in their clinical history, the percentage value of FEV1 was collected.

The variables selected for analysis included demographic variables (age, sex, obesity, smoking, comorbidities, degree of dependence and use of inhaled or oral corticosteroids, antivitamin $\mathrm{K}$ drugs, antiplatelets, statins, ACE inhibitors, Angiotensin-renin blockers); clinical variables on admission (signs and symptoms, laboratory results and radiological findings); treatment received on admission (beta-lactams, macrolides, quinolones, hydroxychloroquine, lopinavir/ritonavir, remdesivir, systemic corticosteroids, immunoglobulins, beta interferon, tocilizumab or anakinra); radiological evolution, ventilatory support (invasive and non-invasive mechanical ventilation and high-flow oxygen therapy) and clinical results (admission to the ICU and death).

Data on patients with COPD were selected and compared to data on non-COPD patents. Factors indicating poor prognosis were analyzed, defined as all-cause mortality. All-cause mortality during hospitalization was the primary endpoint.

For the subsequent data analysis, the STATA statistical system was used.

Qualitative variables are expressed as absolute frequency (n) and percentage (\%). Quantitative variables are expressed as median, interquartile range, and range with a 95\% confidence interval. Qualitative variables were compared using the chi-square test. Continuous variables were compared using the Mann-Whitney test and logistic regression of variables. Statistical significance was defined as a $\mathrm{p}$ value $<0.05$.

\section{Ethical Aspects}

Personal data is processed in strict compliance with Law 14/ 2007, of July 3, on Biomedical Research; Regulation (EU) 2016/679 of the European Parliament and of the Council, of April 27, 2016, on the protection of natural persons with regard to the processing of personal data and the free circulation of said data, and by which repeals Directive 95/46/EC (General Data Protection Regulation); and Organic Law 3/ 2018, of December 5, on the Protection of Personal Data and Guarantee of Digital Rights. The SEMI-COVID-19 Registry was first approved by the Provincial Research Ethics Committee of Malaga (Spain), following the recommendation of the Spanish Agency for Medicines and Health Products (AEMPS). Informed consent was requested from the patients. When it was not possible to obtain it in writing for biosafety reasons or because the patient was already discharged from hospital, verbal informed consent was requested and entered in the medical record.

\section{Results}

Of a total of 10,420 patients included in the SEMI-COVID-19 registry as of May 21, 2020, $746(7.16 \%)$ had a history of COPD. Tables 1 and 2 showed the characteristics of nonCOPD and COPD patients. The data indicated that COPD patients were older than patients without COPD (77 years vs 68 years) and were more likely to be male (82.2\% vs $54.7 \%)$. They had more comorbidities, especially cardiovascular comorbidities (hypertension, hyperlipidemia, diabetes mellitus, atrial fibrillation, heart failure, ischemic heart disease, peripheral vascular disease, renal failure); a higher Charlson Comorbidity Index and age-corrected Charlson Comorbidity Index ( 3 vs 6, p<0.001); and had greater functional dependence. FEV1 was recorded for 364 patients with COPD, with a mean FEV1 of $59 \%$.

The percentage of patients with COPD who were treated with systemic corticosteroids was $7.4 \%$ and the percentage treated with inhaled corticosteroids was $47.2 \%$. Patients with COPD were also more likely to had been taking antiplatelet drugs, anticoagulants, statins, ACE inhibitors, and angiotensin-renin blockers.

In patients with COPD, the clinical presentation of COVID-19 usually involved low-grade fever, dyspnea, and expectoration, with fewer non-respiratory symptoms than non-COPD patients. On examination, these patients 
Table I Characteristics of People with Coronavirus Disease 2019 with or without COPD. Demographic Data and Comorbidity

\begin{tabular}{|c|c|c|c|c|c|}
\hline \multirow[t]{2}{*}{ Characteristics } & \multicolumn{5}{|c|}{ Values are Indicated as the Number (Percentage) or Median (Interquartile Range) } \\
\hline & Overall $(n=10,420)$ & $\mathbf{N}(\%)$ & Non-COPD $(n=9674)$ & COPD $(n=746)$ & $P$ value \\
\hline Median (IQR) age (years) & 10,385 & $69(55-79)$ & $68(54-79)$ & $77(7 \mid-84)$ & - \\
\hline \multicolumn{6}{|l|}{ Age (years): } \\
\hline$<45$ years & 10,385 & $1130(10.9)$ & $1130(11.7)$ & $0(0.0)$ & - \\
\hline $45-55$ years & & $1325(12.8)$ & $1316(13.7)$ & $9(1.2)$ & $<0.001$ \\
\hline 55-65 years & & $1884(18.1)$ & 1798 (18.7) & $86(11.6)$ & $<0.001$ \\
\hline $65-75$ years & & $2258(21.7)$ & $2049(21.3)$ & $209(28.1)$ & 0.596 \\
\hline $75-85$ years & & $2263(21.8)$ & $1973(20.5)$ & $290(39)$ & 0.004 \\
\hline$>85$ years & & 1525 (14.7) & $1376(14.3)$ & $149(20.0)$ & omitted \\
\hline Men & $|0,40|$ & $5893(56.7)$ & $528 I(54.7)$ & $612(82.2)$ & $<0.001$ \\
\hline \multicolumn{6}{|l|}{ Smoking status } \\
\hline Never & 9904 & $6890(69.6)$ & $6783(74)$ & $107(14.7)$ & - \\
\hline Former & & $2482(25.0)$ & $1968(21.4)$ & $514(70.7)$ & $<0.001$ \\
\hline Current & & $532(5.4)$ & $426(4.6)$ & $106(14.6)$ & 0.46 \\
\hline Alcohol use disorder & 10,112 & 470 (4.7) & $384(4.0)$ & $86(11.8)$ & $<0.001$ \\
\hline Obesity (BMI>30) & 4597 & $1537(33.4)$ & $1415(33.6)$ & $122(32.1)$ & 0.57 \\
\hline Hypertension & 10,404 & $5242(50.4)$ & 4729 (48.9) & $513(69.1)$ & $<0.001$ \\
\hline Hyperlipidemia & $|0,40|$ & 4078 (39.2) & $3682(38.1)$ & $396(53.4)$ & $<0.001$ \\
\hline Diabetes Mellitus & 10,379 & $1936(18.7)$ & $1745(18.1)$ & $191(25.8)$ & $<0.001$ \\
\hline Anxiety disorder & 10,377 & $842(8.1)$ & $779(8.0)$ & $63(8.5)$ & 0.71 \\
\hline Depression & 10,376 & $1092(10.5)$ & $1014(10.5)$ & $78(10.5)$ & 0.99 \\
\hline Atrial fibrillation & 10,395 & $1175(11.3)$ & $1000(10.4)$ & $175(23.6)$ & $<0.001$ \\
\hline Myocardial infarction & 10,410 & $619(5.6)$ & $521(5.4)$ & $98(13.2)$ & $<0.001$ \\
\hline Heart failure & 10,406 & $775(7.5)$ & $643(6.7)$ & $132(17.8)$ & $<0.001$ \\
\hline Transient ischemic attack & 10,377 & $747(7.2)$ & $657(6.8)$ & $90(12.2)$ & $<0.001$ \\
\hline Dementia & 10,403 & $1066(10.3)$ & $987(10.2)$ & $79(10.6)$ & 0.74 \\
\hline Neurodegenerative disease & $|0,40|$ & $979(9.4)$ & $908(9.4)$ & 7I (9.5) & 0.89 \\
\hline Peripheral arterial disease & 10,397 & $503(4.8)$ & $414(4.3)$ & $89(12.0)$ & $<0.001$ \\
\hline Mild chronic liver disease & 10,393 & $294(2.8)$ & $255(2.6)$ & $39(5.2)$ & $<0.001$ \\
\hline Moderate-severe chronic liver disease & $|0,40|$ & $110(1.0)$ & $90(0.9)$ & $20(2.7)$ & $<0.001$ \\
\hline Chronic kidney disease & 10,399 & $631(6.1)$ & $549(5.7)$ & $82(11.0)$ & $<0.001$ \\
\hline Dialysis & 10,352 & $16(0.2)$ & $14(0.2)$ & $2(0.3)$ & 0.64 \\
\hline Cancer & 10,388 & $845(8.1)$ & $740(7.7)$ & $105(14.1)$ & $<0.001$ \\
\hline Obstructive Sleep Apnea Syndrome & 10,337 & $654(6.3)$ & $524(5.5)$ & $130(17.6)$ & $<0.001$ \\
\hline \multicolumn{6}{|l|}{ Dependency status } \\
\hline Independent & 10,250 & $8519(83.1)$ & 7960 (83.7) & $559(76.7)$ & - \\
\hline Moderate dependency & & $984(9.6)$ & $856(9.0)$ & $128(17.4)$ & $<0.001$ \\
\hline Severe dependency & & $747(7.3)$ & $700(7.4)$ & $47(6.4)$ & 0.76 \\
\hline Charlson Comorbidity Index & 10,084 & I (0-2) & I (0-2) & $2(1-4)$ & $<0.001$ \\
\hline Charlson Comorbidity Index, age corrected & 10,048 & $3(1-5)$ & $3(1-5)$ & $6(4-8)$ & $<0.001$ \\
\hline
\end{tabular}

were more likely to present with hypoxemia, tachypnea, and confusional symptoms, with the presence of rhonchi and wheezing on pulmonary auscultation.

All-cause mortality in COPD patients was $38.3 \%$, compared to $19.2 \%$ in non-COPD patients $(\mathrm{p}<0.001)$. Male sex, a history of hypertension, heart failure, moderate-severe chronic kidney disease, the presence of cerebrovascular disease with sequelae, degenerative neurological disease, dementia, functional dependence, and a higher Charlson Comorbidity Index had been associated with higher mortality in COPD patients (Tables 3 and 4). The relationship was nearly significant in patients with a history of myocardial 
Table 2 Characteristics of People with Coronavirus Disease 2019 with or without COPD. Clinical Data and Evolution

\begin{tabular}{|c|c|c|c|c|c|}
\hline \multirow[t]{2}{*}{ Characteristics } & \multicolumn{5}{|c|}{ Values are Indicated as the Number (Percentage) or Median (Interquartile Range) } \\
\hline & Overall $(n=10,420)$ & $\mathbf{N}(\%)$ & Non-COPD ( $n=9674)$ & COPD $(n=746)$ & P value \\
\hline \multicolumn{6}{|l|}{ Habitual treatment } \\
\hline Immunosuppressants & 10,383 & $362(3.5)$ & $335(3.5)$ & $27(3.6)$ & 0.82 \\
\hline Systemic corticosteroids & 10,392 & $450(4.3)$ & $395(4.0)$ & $55(7.4)$ & $<0.001$ \\
\hline Inhaled corticosteroids & 10,340 & $1031(10.0)$ & $683(7.1)$ & $348(47.2)$ & $<0.001$ \\
\hline \multicolumn{6}{|l|}{ Anticoagulants } \\
\hline None & 10,338 & $9148(88.5)$ & $8582(89.4)$ & $566(76.4)$ & - \\
\hline Antivitamin $\mathrm{K}$ & & $653(6.3)$ & $552(5.8)$ & $101(13.6)$ & $<0.001$ \\
\hline DOACs & & $456(4.4)$ & $388(4.0)$ & $68(9.2)$ & $<0.001$ \\
\hline LMWH & & $81(0.8)$ & $75(0.8)$ & $6(0.8)$ & 0.65 \\
\hline Antiplatelet drugs & 10,319 & $159 \mid(15.4)$ & $1393(14.5)$ & $198(26.9)$ & $<0.001$ \\
\hline Statins & 10,319 & $3302(32.0)$ & $2928(30.6)$ & $374(50.6)$ & $<0.001$ \\
\hline ACE inhibitors & 10,330 & $1757(17.0)$ & $1590(16.6)$ & $167(22.6)$ & $<0.001$ \\
\hline Angiotensin-renin blockers & 10,334 & $2014(19.5)$ & $1829(19)$ & $185(25)$ & $<0.001$ \\
\hline \multicolumn{6}{|l|}{ Symptoms and vital signs } \\
\hline Temperature $>38^{\circ} \mathrm{C}$ & 10,369 & $6581(63.5)$ & $6181(64.2)$ & $400(53.8)$ & $<0.001$ \\
\hline Low-grade fever & 10,369 & $2195(21.2)$ & $2025(2 \mathrm{I})$ & $170(22.9)$ & $<0.001$ \\
\hline Dyspnea & 10,369 & $5939(53.4)$ & $5402(56.2)$ & $537(72.3)$ & $<0.001$ \\
\hline Dry cough & 10,368 & $6084(58.7)$ & $5724(59.5)$ & $360(48.5)$ & $<0.001$ \\
\hline Cough with expectoration & & $1652(15.9)$ & $1452(15.1)$ & $200(26.9)$ & $<0.001$ \\
\hline Fatigue & 10,157 & $4493(44.2)$ & $4193(44.5)$ & $300(4 \mid .2)$ & 0.088 \\
\hline Anorexia & 10,102 & $2045(20.2)$ & $1903(20.3)$ & $142(19.6)$ & 0.68 \\
\hline Myalgia & 10,198 & $3146(30.9)$ & $2969(31.4)$ & $177(24.2)$ & $<0.001$ \\
\hline Headache & 10,160 & $1192(11.7)$ & $1152(12.2)$ & $40(5.5)$ & $<0.001$ \\
\hline Diarrhea & 10,287 & $2339(22.7)$ & $2239(23.5)$ & $100(13.6)$ & $<0.001$ \\
\hline Nausea & 10,121 & $1274(12.6)$ & $1233(13.1)$ & $4 \mid(5.7)$ & $<0.001$ \\
\hline Vomiting & 10,249 & $759(7.4)$ & $740(7.8)$ & $19(2.6)$ & $<0.001$ \\
\hline Abdominal pain & 10,235 & $683(6.7)$ & $649(6.8)$ & $34(4.6)$ & 0.022 \\
\hline Ageusia & 9971 & $656(6.6)$ & $632(6.8)$ & $24(3.4)$ & $<0.001$ \\
\hline Anosmia & 9967 & $584(5.9)$ & $563(6.1)$ & $21(3.0)$ & 0.001 \\
\hline Sore throat & 10,147 & $994(9.8)$ & $926(9.8)$ & $68(9.4)$ & 0.68 \\
\hline \multicolumn{6}{|l|}{ Vital signs at triage } \\
\hline Temperature $>38^{\circ} \mathrm{C}$ & 9978 & $1611(16.2)$ & $1516(16.4)$ & $95(13.3)$ & 0.029 \\
\hline Temperature, median (IQR) ${ }^{\circ} \mathrm{C}$ & 9978 & $37(36.4-37.8)$ & $36.9(36.2-37.7)$ & $37(36.4-37.8)$ & 0.004 \\
\hline Sat $<90 \%$ & 10,084 & $2211(21.9)$ & $1928(20.6)$ & $283(38.8)$ & $<0.001$ \\
\hline Sat02\% Median (IQR) & 10,084 & $94(9 \mid-97)$ & $95(91-97)$ & $92(88-95)$ & $<0.001$ \\
\hline Respiratory rate $>20$ breaths/min & 10,097 & $3 \mid 45(31.2)$ & $2797(29.9)$ & $348(47.9)$ & $<0.001$ \\
\hline Heart rate $>100$ beats $/ \mathrm{min}$ & 9990 & $2181(21.8)$ & $2036(22.0)$ & $145(20.0)$ & 0.22 \\
\hline Heart rate Median (IQR) & 9990 & $87(76-100)$ & $87(76-100)$ & $85(75-98)$ & 0.08 \\
\hline SBP, median (IQR) mmHg & 9870 & $127(|| 4-|4|)$ & $127(115-140)$ & $127(113-144)$ & 0.96 \\
\hline DBP, median (IQR) mmHg & 9860 & $74(65-82)$ & $74(65-82)$ & $72(61-80)$ & $<0.001$ \\
\hline Confusion & 10,270 & $1227(12.0)$ & $1100(11.5)$ & $127(17.3)$ & $<0.001$ \\
\hline Wheezing & 10,105 & $625(6.2)$ & $50 \mid(5.3)$ & $124(17.3)$ & $<0.001$ \\
\hline Rhonchi & 10,097 & $1088(10.8)$ & $890(9.5)$ & $198(27.6)$ & $<0.001$ \\
\hline Crackles & 10,107 & $5324(52.7)$ & $4937(52.6)$ & $387(53.9)$ & 0.49 \\
\hline \multicolumn{6}{|l|}{ Evolution } \\
\hline Non-invasive mechanical ventilation & 10,327 & $503(4.9)$ & $429(4.5)$ & $74(10.1)$ & $<0.001$ \\
\hline High-flow nasal cannula & 10,264 & $832(8.1)$ & $736(7.7)$ & $96(13.2)$ & $<0.001$ \\
\hline
\end{tabular}


Table 2 (Continued).

\begin{tabular}{|c|c|c|c|c|c|}
\hline \multirow[t]{2}{*}{ Characteristics } & \multicolumn{5}{|c|}{ Values are Indicated as the Number (Percentage) or Median (Interquartile Range) } \\
\hline & Overall $(n=10,420)$ & $\mathbf{N}(\%)$ & Non-COPD (n=9674) & COPD $(n=746)$ & $P$ value \\
\hline Invasive mechanical ventilation & 10,326 & $663(6.4)$ & $627(6.5)$ & $36(4.9)$ & 0.083 \\
\hline Prone position & 10,307 & $1020(9.9)$ & $938(9.8)$ & $82(11.2)$ & 0.21 \\
\hline ICU admission & 10,400 & $841(8.1)$ & $788(8.2)$ & $53(7.1)$ & 0.31 \\
\hline Hospital stay in days, median (IQR) & $9(5-14)$ & & $9(5-14)$ & $10(6-16)$ & 0.80 \\
\hline Mortality & $2142(20.6)$ & & $1856(19.2)$ & $286(38.3)$ & $<0.0001$ \\
\hline
\end{tabular}

infarction and obesity. Associating the comorbidities in clusters: risk factors, neurological, cardiovascular, hepaticrenal and oncological, we found that cardiovascular and hepatic-renal were associated with an increase in mortality in COPD patients with COVID. COPD patients with SARSCoV2 infection who presented some comorbid pathology had higher mortality than those who did not have any comorbidity (Table 5).

Higher all-cause mortality was observed in patients in regular treatment with systemic corticosteroids and in patients who were anticoagulated and antiaggregated with acetylsalicylic acid, which could possibly reflect increased cardiovascular disease. However, neither chronic treatment with ACE inhibitors nor with angiotensin-renin blockers were associated with higher all-cause mortality in this population.

We found no differences in survival in COPD patients among current smokers, former smokers, and never smokers (Figure 1). A worse FEV1 value also did not correlate with mortality.

In regard to hemogram parameters, all- cause mortality was associated with higher levels of leukocytes (7180 vs 6400, p 0.002), neutrophils (5125 vs 4640, p 0.004), C-reactive protein (CRP) (99.2 vs 63.9, p<0.001), creatinine (1.21 vs $1.0, \mathrm{p} 0.001)$, lactate dehydrogenase (LDH) (352 vs $270, \mathrm{p}<0.001)$, procalcitonin (0.2 vs 0.1 , p 0.003$)$, and D-Dimer (1014 vs 695, p 0.002).

Radiologically, the presence of bilateral condensation, bilateral interstitial infiltrates, and radiological worsening at one week was associated with an increased mortality rate.

In terms of treatment, $56.9 \%$ of COPD patients received treatment with lopinavir/ritonavir and 49\% received systemic corticosteroids; greater survival was not observed among these patients. Survival was higher among patients with COPD who were treated with hydroxychloroquine $(87.1 \%$ vs $74.9 \%, p<0.001)$ and with macrolides $(57.9 \%$ vs $50 \%, \mathrm{p}<0.037)$. An improved prognosis was not observed with the use of beta-lactams or quinolones.
Neither prone positioning, non-invasive mechanical ventilation, high-flow nasal cannula, or invasive mechanical ventilation were associated with a better prognosis.

\section{Discussion}

This is the first work that describes the characteristics and prognosis of COPD patients hospitalized with SARS-CoV-2 infection.

One of the most notable findings among these data is the low prevalence of COPD patients. Given that COVID-19 is a viral disease with respiratory involvement, it would be logical to expect to find a higher percentage of COPD patients. In previous works, the incidence of COPD in patients hospitalized with COVID-19 has been estimated to be $0.95 \%{ }^{3}$ and the prevalence of patients with COVID-19 who had COPD has been estimated to be $2 \% .{ }^{10}$ In previously published data from the SEMI-COVID-19 registry, the incidence of COPD was $7.7 \% .^{12}$ The prevalence found in our registry is higher than in the New York area series $(5.4 \%)$, but lower than some of the Chinese series. ${ }^{13}$

One of the reasons can explain the low prevalence of COVID-19 among COPD patients could be the use of drugs for respiratory disease like inhaled steroids, betaagonists or anticholinergics, specially tiotropium. ${ }^{14}$ In the other side, glycopyrronium and formoterol have been shown to reduce cellular susceptibility to coronavirus infection in vitro. They do so by inhibiting the expression of coronavirus receptors, reducing endosomal activity, and modulating the inflammatory responses induced by it in the airway. Indeed, basic treatment for COPD patients usually includes long-acting muscarinic antagonists (LAMA) and/or long-acting b2-agonists (LABA). ${ }^{15}$ Based on this low prevalence, it could be said that COPD patients would not have an increased risk of contracting SARS-CoV2 infection. These data are taken from hospitalized patients, and to be able to affirm these results 
Table 3 Mortality of COPD Patients with Coronavirus Disease 2019 According to Demographic Data and Antecedents

\begin{tabular}{|c|c|c|c|c|c|}
\hline \multicolumn{6}{|c|}{ Values are Indicated as the Number (Percentage) or Median (Interquartile Range) } \\
\hline Characteristics & Overall $(n=746)$ & Survivors $(n=460)$ & Deceased $(n=286)$ & Odds Ratio $(95 \% \mathrm{Cl})$ & $P$ value \\
\hline Age (years) & $77(7 \mid-84)$ & $75(66-82)$ & $79(74-86)$ & $1.06(1.04-1.08)$ & $<0.001$ \\
\hline Men & $612(82.2)$ & $365(79.4)$ & $247(86.7)$ & $1.69(1.12-2.55)$ & 0.012 \\
\hline \multicolumn{6}{|l|}{ Comorbidities } \\
\hline \multicolumn{6}{|l|}{ FEVI } \\
\hline$<30$ & $21(5.8)$ & II (4.9) & $10(7.3)$ & $1.74(0.58-5.26)$ & 0.33 \\
\hline $30-49$ & $84(23.1)$ & $52(22.9)$ & $32(23.4)$ & $1.18(0.52-2.69)$ & 0.70 \\
\hline 50-79 & $224(61.5)$ & $|4|(62.1)$ & $83(60.6)$ & $1.13(0.53-2.39)$ & 0.75 \\
\hline$\geq 80$ & $35(9.6)$ & $23(10.1)$ & $12(8.8)$ & Ref. (I) & - \\
\hline FEVI $<50$ & $105(28.8)$ & $63(27.8)$ & $42(30.7)$ & I (ref.) & - \\
\hline FEVI $>50$ & $259(7 \mid .2)$ & $164(72.2)$ & $95(69.3)$ & $0.87(0.55-1.38)$ & 0.55 \\
\hline \multicolumn{6}{|l|}{ Smoking status } \\
\hline Never & $107(14.8)$ & $59(13.3)$ & $48(17.0)$ & I (ref.) & - \\
\hline Former & $514(70.7)$ & $316(71.0)$ & $198(70.2)$ & $0.77(0.51-1.17)$ & 0.22 \\
\hline Current & $106(14.6)$ & $70(15.7)$ & $36(12.8)$ & $0.63(0.36-1.1)$ & 0.12 \\
\hline Obesity (BMI>30) & $122(32.1)$ & $73(30.7)$ & $49(34.5)$ & $1.19(0.77-1.85)$ & 0.44 \\
\hline Hypertension & $513(69.1)$ & $298(65.0)$ & $215(75.7)$ & $1.67(1.2-2.3)$ & 0.002 \\
\hline Hyperlipidemia & $396(53.4)$ & $239(52.2)$ & $157(55.3)$ & $1.13(0.84-1.52)$ & 0.41 \\
\hline Diabetes mellitus & $191(25.8)$ & $119(26.1)$ & $72(25.4)$ & $0.96(0.69-1.35)$ & 0.82 \\
\hline Atrial fibrillation & $175(23.6)$ & $103(22.5)$ & $72(25.4)$ & $1.18(0.83-1.66)$ & 0.36 \\
\hline Myocardial infarction & $98(13.2)$ & $52(11.3)$ & $46(16.1)$ & 1.51 (0.98-2.32) & 0.059 \\
\hline Heart failure & I32 (I7.8) & $58(12.7)$ & $74(26.1)$ & $2.43(1.65-3.56)$ & $<0.001$ \\
\hline Transient ischemic attack & $90(12.2)$ & $48(10.5)$ & $42(15.1)$ & $1.52(0.97-2.37)$ & 0.066 \\
\hline Dementia & $79(10.6)$ & $36(7.8)$ & $43(15.0)$ & $2.08(1.3-3.33)$ & 0.002 \\
\hline Neurodegenerative disease & $71(9.5)$ & $34(7.4)$ & $37(12.9)$ & $1.85(1.13-3.03)$ & 0.014 \\
\hline Peripheral arterial disease & $89(12.0)$ & $5 I(I I . I)$ & $38(13.4)$ & $1.24(0.79-1.94)$ & 0.35 \\
\hline Chronic kidney disease & $82(I 1.0)$ & $4 \mid(8.9)$ & $4 \mid(14.4)$ & $1.71(1.08-2.72)$ & 0.022 \\
\hline Cancer & $105(14.1)$ & $60(13.1)$ & $45(15.8)$ & $1.25(0.82-1.89)$ & 0.30 \\
\hline Obstructive Sleep Apnea Syndrome & $130(17.6)$ & $83(18.1)$ & $47(16.9)$ & $0.92(0.62-1.34)$ & 0.66 \\
\hline \multicolumn{6}{|l|}{ Dependency status } \\
\hline Independent & $559(76.2)$ & $377(82.7)$ & $182(65.5)$ & I (ref.) & - \\
\hline Moderate dependency & $128(17.4)$ & $61(13.4)$ & $67(24.1)$ & $2.28(1.54-3.36)$ & $<0.001$ \\
\hline Severe dependency & $47(6.4)$ & $18(4.0)$ & $29(10.4)$ & $3.34(1.81-6.17)$ & $<0.001$ \\
\hline Charlson Comorbidity Index & $2(1-4)$ & $2(1-4)$ & $3(2-5)$ & $1.17(1.09-1.26)$ & $<0.001$ \\
\hline Charlson Comorbidity Index, age corrected & $6(4-8)$ & $5(4-7)$ & $6(5-9)$ & $1.23(1.15-1.32)$ & $<0.001$ \\
\hline \multicolumn{6}{|l|}{ Habitual treatment } \\
\hline Immunosuppressants & $27(3.6)$ & $19(4.1)$ & $8(2.8)$ & $0.68(0.29-1.56)$ & 0.36 \\
\hline Systemic corticosteroids & $55(7.4)$ & $27(5.9)$ & $28(9.9)$ & $1.75(1.01-3.04)$ & 0.046 \\
\hline Inhaled corticosteroids & $348(47.2)$ & $216(47.2)$ & $132(47.1)$ & I $(0.74-\mid .35)$ & 0.996 \\
\hline Antivitamin $\mathrm{K}$ & $101(13.6)$ & $48(10.5)$ & $53(18.7)$ & $1.94(1.27-2.98)$ & 0.002 \\
\hline Antiplatelet Drugs & $198(26.9)$ & $105(23.0)$ & $93(33.2)$ & $1.67(1.20-2.32)$ & 0.002 \\
\hline Statins & $374(50.6)$ & $237(51.8)$ & $137(48.8)$ & $0.89(0.66-1.19)$ & 0.43 \\
\hline ACE In & $167(22.6)$ & $101(22.1)$ & $66(23.5)$ & $1.09(0.76-1.54)$ & 0.65 \\
\hline Angiotensin-renin blockers & $185(25)$ & $107(23.3)$ & $78(27.7)$ & $1.26(0.90-1.77)$ & 0.19 \\
\hline
\end{tabular}

we would also need to have data from non-hospitalized COPD patients.

However, COPD patients with COVID-19 have a poor prognosis. COPD patients are older and have more comorbidities, especially cardiovascular comorbidities. This association between COPD and comorbidity has previously been described, especially in patients hospitalized for an exacerbation, which is an important prognostic 
Table 4 Mortality of COPD Patients with Coronavirus Disease 2019 According to Clinical Data and Treatment

\begin{tabular}{|c|c|c|c|c|c|}
\hline \multicolumn{6}{|c|}{ Values are Indicated as the Number (Percentage) or Median (Interquartile Range) } \\
\hline Characteristics & Overall $(n=746)$ & Survivors $(n=460)$ & Deceased $(n=286)$ & Odds Ratio (95\% Cl) & $P$ value \\
\hline \multicolumn{6}{|l|}{ Symptoms and vital signs } \\
\hline Dyspnea & $537(72.3)$ & $304(66.1)$ & $233(82.3)$ & $2.39(1.67-3.43)$ & $<0.001$ \\
\hline Temperature $>38^{\circ} \mathrm{C}$ & $95(13.3)$ & $47(10.6)$ & $48(17.5)$ & $1.78(1.15-2.74)$ & 0.009 \\
\hline Oxygen saturation $<90 \%$ & $283(38.8)$ & $13 \mid(29.1)$ & $152(54.3)$ & $0.35(0.25-0.47)$ & $<0.001$ \\
\hline Oxygen saturation \% Median (IQR) & $92(88-95)$ & $93(90-96)$ & $90(86-94)$ & $0.91(0.88-0.93)$ & $<0.001$ \\
\hline Respiratory rate $>20$ breaths $/ \mathrm{min}$ & $348(47.9)$ & $169(37.6)$ & $179(64.6)$ & $3.03(2.22-4.13)$ & $<0.001$ \\
\hline \multicolumn{6}{|l|}{ Heart rate } \\
\hline$>100$ beats $/ \mathrm{min}$ & $145(20.0)$ & $80(17.9)$ & $65(23.4)$ & $1.40(0.97-2.02)$ & 0.073 \\
\hline Median (IQR) & 85 (75-98) & 85 (74-98) & $86(76-100)$ & $1.01(0.99-1.01)$ & 0.18 \\
\hline SBP, median (IQR) mmHg & $127(113-144)$ & $129(116-144)$ & $125(|10-14|)$ & $0.99(0.99-1)$ & 0.09 \\
\hline DBP, median (IQR) $\mathrm{mmHg}$ & $72(61-80)$ & $73(64-8 I)$ & $70(60-80)$ & $0.99(0.98-0.99)$ & 0.008 \\
\hline Confusion & $127(17.3)$ & $53(11.7)$ & $74(26.3)$ & $2.71(1.83-4)$ & $<0.001$ \\
\hline \multicolumn{6}{|l|}{ Radiological findings } \\
\hline At admission & & & & & \\
\hline Bilateral condensation & $203(27.7)$ & $107(23.7)$ & $96(33.9)$ & $1.8(1.29-2.6)$ & 0.001 \\
\hline Bilateral interstitial infiltrates & $359(48.9)$ & $202(44.7)$ & $157(55.7)$ & $1.6(1.13-2.15)$ & 0.006 \\
\hline \multicolumn{6}{|l|}{ Progress (7 days) } \\
\hline Bilateral condensation & $198(36.5)$ & II4 (30.7) & $84(49.1)$ & $2.38(1.58-3.57)$ & $<0.001$ \\
\hline Bilateral interstitial infiltrates & $316(58.1)$ & $201(53.9)$ & $115(67.3)$ & $1.55(1.03-2.32)$ & 0.035 \\
\hline Radiological worsening & $277(50.8)$ & $147(39.6)$ & $130(74.7)$ & $4.5(3.02-6.72)$ & $<0.001$ \\
\hline \multicolumn{6}{|l|}{ Treatment } \\
\hline Lopinavir/ritonavir & 421 (56.9) & $267(58.4)$ & $154(54.4)$ & $0.85(0.63-1.45)$ & 0.29 \\
\hline Interferon-beta & $94(12.8)$ & $4 \mid(9.0)$ & $53(19.0)$ & $2.36(1.52-3.66)$ & $<0.001$ \\
\hline Remdesivir & $4(0.6)$ & $2(0.4)$ & $2(0.7)$ & $1.66(0.23-11.85)$ & 0.61 \\
\hline Hydroxychloroquine & $610(82.4)$ & $398(87.1)$ & $212(74.9)$ & $0.44(0.3-0.65)$ & $<0.001$ \\
\hline Chloroquine & $26(3.5)$ & $16(3.5)$ & $10(3.6)$ & $1.02(0.46-2.29)$ & 0.96 \\
\hline Colchicine & $10(1.4)$ & $4(0.9)$ & $6(2.2)$ & $2.5(0.7-8.9)$ & 0.16 \\
\hline Tocilizumab & $50(6.8)$ & $23(5.0)$ & $27(9.6)$ & $1.99(1.12-3.56)$ & 0.019 \\
\hline Immunoglobulin & $2(0.3)$ & $\mathrm{I}(0.2)$ & $\mathrm{I}(0.4)$ & $1.7(0.1-26-6)$ & 0.72 \\
\hline Anakinra & $4(0.6)$ & $3(0.7)$ & I $(0.4)$ & $0.54(0.06-5.24)$ & 0.60 \\
\hline Systemic corticosteroids & $364(49.3)$ & $215(47.1)$ & $149(53.0)$ & $1.27(0.94-I .7 I)$ & 0.12 \\
\hline Beta-lactams & $561(76.3)$ & $329(72.3)$ & $232(82.9)$ & $1.85(1.28-2.69)$ & 0.001 \\
\hline Macrolides & $400(55.0)$ & $263(57.9)$ & $137(50.0)$ & $0.73(0.54-0.98)$ & 0.037 \\
\hline Quinolones & $134(18.5)$ & $78(17.2)$ & $56(20.5)$ & $1.24(0.85-1.82)$ & 0.27 \\
\hline Non-invasive mechanical ventilation & $74(10.1)$ & $34(7.5)$ & $40(14.4)$ & $2.09(1.29-3.40)$ & 0.003 \\
\hline High-flow nasal cannula & $96(13.2)$ & $46(10.2)$ & $50(18.0)$ & $1.93(1.25-2.97)$ & 0.003 \\
\hline Invasive mechanical ventilation & $36(4.9)$ & $8(1.8)$ & $28(10.1)$ & $6.27(2.82-13.97)$ & $<0.001$ \\
\hline Prone position & $82(11.2)$ & $25(5.5)$ & $57(20.6)$ & $4.44(2.70-7.29)$ & $<0.001$ \\
\hline Hospital stay in days, median (IQR) & $10(6-16)$ & $12(7-17)$ & $7(3-12)$ & $0.95(0.93-0.96)$ & $<0.001$ \\
\hline
\end{tabular}

factor. Almagro confirmed the elevated prevalence of associated diseases in patients with COPD who are admitted to the Spanish Internal Medicine Services; in a later article he showed that mortality at 3 months in hospitalized COPD patients was associated with comorbidities both measured with the Charlson index and total of comorbidities. ${ }^{2,16}$
Roberts demonstrated that comorbidities adversely affect in hospitalized COPD exacerbations. ${ }^{17}$ Alqahtani in a systematic review and meta-analysis found that heart failure, renal failure, depression and alcohol use were all associated with an increased risk of 30-day all-cause readmission. $^{18}$ The association between comorbidities 
Table 5 Comorbidity and mortality of COPD Patients with Coronavirus Disease 2019

\begin{tabular}{|c|c|c|c|c|c|}
\hline Characteristics & Overall $(n=746)$ & Survivors $(n=460)$ & Deceased $(n=286)$ & Odds Ratio (95\% Cl) & $P$ value \\
\hline \multicolumn{6}{|l|}{ Comorbidity - CVRF } \\
\hline No & $169(22.6)$ & III (24.I) & $58(20.3)$ & I (ref.) & - \\
\hline Yes & $577(77.4)$ & $349(75.9)$ & $228(79.7)$ & $1.25(0.87-1.79)$ & 0.22 \\
\hline \multicolumn{6}{|l|}{ Comorbidity - NRL ${ }^{\mathrm{b}}$} \\
\hline No & $583(78.2)$ & $373(81.1)$ & $210(73.4)$ & I (ref.) & - \\
\hline Yes & $163(21.9)$ & $87(18.9)$ & $76(26.6)$ & $1.55(1.09-2.20)$ & 0.014 \\
\hline \multicolumn{6}{|l|}{ Comorbidity - Cardio ${ }^{c}$} \\
\hline No & $406(54.4)$ & $266(57.8)$ & $140(49.0)$ & I (ref.) & - \\
\hline Yes & $340(45.6)$ & $194(42.2)$ & I $46(52.0)$ & $1.43(1.06-1.92)$ & 0.018 \\
\hline \multicolumn{6}{|l|}{ Comorbidity - K.L ${ }^{d}$} \\
\hline No & $614(82.3)$ & $387(84.1)$ & $227(79.4)$ & I (ref.) & - \\
\hline Yes & $132(17.7)$ & $73(15.9)$ & $59(20.6)$ & $1.38(0.94-2.02)$ & 0.10 \\
\hline \multicolumn{6}{|l|}{ Comorbidity - Onco ${ }^{\mathrm{e}}$} \\
\hline No & $623(83.5)$ & $392(85.2)$ & $231(80.8)$ & I (ref.) & - \\
\hline Yes & $123(16.5)$ & $68(14.8)$ & $55(19.2)$ & $1.37(0.93-2.03)$ & 0.11 \\
\hline \multicolumn{6}{|l|}{ Comorbidity $^{f}$} \\
\hline No & $64(8.6)$ & $51(11.1)$ & $13(4.5)$ & I (ref.) & - \\
\hline Yes & $682(91.4)$ & 409 (88.9) & $273(95.5)$ & $2.62(\mid .40-4.91)$ & 0.003 \\
\hline
\end{tabular}

Notes: ${ }^{a}$ Comorbidity-CVRF: Considering as comorbidity the presence of any of the following Cardiovascular Risk Factors: HBP, Dyslipidemia, Diabetes Mellitus (with or without target organ injury) or Obesity. ${ }^{\mathrm{b}}$ Comorbidity-NRL: We consider as comorbidity the presence of any of the following pathologies: Dementia, Neurodegenerative

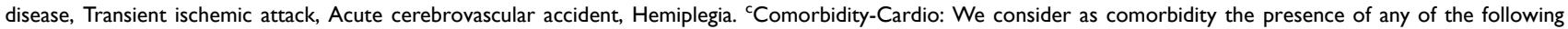
pathologies: Atrial fibrillation, myocardial infarction, Angor, CHF, Peripheral vascular disease. ${ }^{\mathrm{d}}$ Comorbidity-K.L: We consider as comorbidity the presence of any of the following pathologies: chronic kidney disease or Hemodialysis, mild or moderate-severe chronic liver disease. ${ }^{\circ}$ Comorbidity-Onco: We consider as comorbidity the presence of any of the following pathologies: solid neoplasia with or without metastasis, leukemia or lymphoma. ${ }^{\mathrm{f}}$ Comorbidity: In this case we consider as comorbidity the presence of any of the pathologies included in the previous ones.

and the severity of SARS-CoV-2 infection has also been described, finding that greater severity is correlated with increased comorbidity. Thus, the presence of two or more comorbidities was observed more frequently in severe cases than in non-severe cases $(40.0 \%$ vs $29.4 \%) .{ }^{4}$ In our work, the Charlson Comorbidity Index, a comorbidity index that has been validated as a prognostic factor in

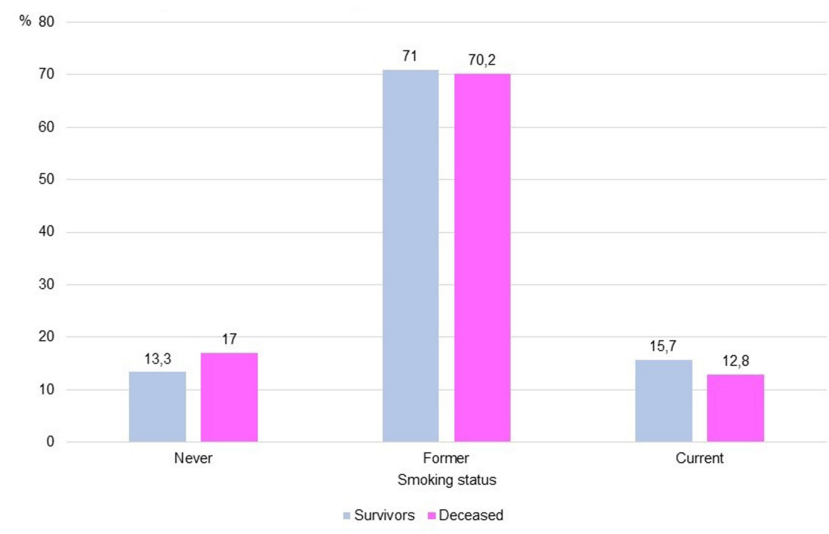

Figure I Mortality according to smoking status in COPD with COVID-19.
COPD, is also associated with a worse prognosis in patients with COVID-19. Although obesity is considered a risk factor in COVID, in our work it is not related to mortality in COPD patients. ${ }^{19}$ This may be justified by a protective effect of obesity on all-cause mortality in COPD patients, described in several studies. This protection is more evident for subjects with a lower FEV1. ${ }^{20}$ Several mechanisms have been proposed that may explain why COVID-19 is more frequent in male patients, elderly with multimorbidity. The higher incidence in men may be associated with the fact that androgen receptor activity is required for the transcription of the TMPRSS2 gene.

In old age there is multisystem dysregulation with a reduced physiological reserve and a poor immune response. In an aging immune system there is a chronic low systemic inflammatory state with elevated levels of IL-6 and C-reactive protein and increased susceptibility to infection. In diabetes there is a low-grade systemic inflammation, which can facilitate a greater release of cytokines and an altered immune response after infection. The pancreas expresses ECA2, through which the coronavirus can 
enter the islets and cause acute B-cell dysfunction, leading to acute hyperglycemia. Many of the poor prognostic comorbidities of COViD-19 share insulin resistance. Multimorbidity is also associated with elevated plasminogen levels. Plasmin and other proteases can cleave a newly inserted furin site in the SARS-CoV-2 protein S, increasing its infectivity and virulence. ${ }^{21}$

Some coronaviruses have been associated with exacerbations of COPD, but neither MERS-CoV nor SARS-CoV -1 showed this association. ${ }^{22,23}$ The presentation of COVID-19 in patients with COPD has been described as different from an exacerbation of COPD, since they present with flu-like symptoms such as fever, anorexia, myalgia, and gastrointestinal symptoms. ${ }^{24}$ However, in the patients analyzed in this study, the usual presentation included increased dyspnea and expectoration and the presence of rhonchi and wheezing on auscultation, similar to a normal infectious exacerbation. Based on this, we recommend performing a SARS-CoV-2 test in all COPD patients with symptoms of exacerbation.

In our series, as in others, COPD patients hospitalized for COVID-19 have a high mortality rate and COPD is considered a predictor of poor prognosis. In the work by Wang et al, patients with COPD were found to be 5.9 times more at risk of suffering severe forms of COVID19 than patients without COPD. ${ }^{25}$ In the work by Zhao et al, the presence of COPD entailed a four-fold risk of suffering a serious course of COVID-19. ${ }^{9}$ Alqahtani et al indicate that the presence of COPD increased the risk of severe coronavirus, with a RR of 1.88 . They found that $63 \%$ of patients with COPD developed severe forms of the disease compared to $33.4 \%$ of those without COPD, with a mortality rate of $60 \%$ among patients with COPD. ${ }^{10}$ In patients older than age 60 who are diagnosed with COVID-19, COPD was a predictor of death, (OR $2.24){ }^{26}$ In a meta-analysis, COPD was associated with an increased mortality risk, (OR 3.53). ${ }^{27}$ In the work by Lippi, COPD was significantly associated with severe COVID-19, OR: $5.69{ }^{28}$ COPD has also been described as a risk factor for disease progression (HR 2.01, 95\% CI 1.38-2.93). ${ }^{29}$ It is possible that poor prognosis depends only on the COPD itself or it may be partially associated with the multimorbidity of these patients.

Smokers are at higher risk of contracting respiratory tract infections such as influenza, and that these are more serious, as in the previous MERS outbreak where smokers had a higher mortality. A low prevalence of smoking has been found in COVID-19 cases, although published data on the severity of COVID-19 in smokers is variable. In a meta-analysis, active smokers were found to be 1.45 times more likely to have serious complications compared to ex-smokers and those who had never smoked. They also had a higher mortality rate of $38.5 \%$. In another work, the combined OR was 2.20. However, in other studies, the association between active smoking and severe COVID-19 was not found to be significant. It has even been reported that smoking may be associated with a non-significant trend towards decreased severity of the disease and perhaps even a protective factor against disease progression (HR 0.56, 95\% CI 0.34 at 0.91). Vardavas in a systematic review of studies concludes that it is very likely that smoking is associated with poor progression and poor prognosis of COVID-19.

It has been postulated that the infection is more severe in patients with COPD and in smokers because exposure to tobacco causes an alteration in the regulation of ACE-2 expression, these patients presenting an overexpression of ACE-II, the receptor of the virus to enter the cell; furthermore, the expression levels of ACE-II are inversely related to FEV1. However, in our study, the smoking status has not been related to all-cause mortality. It is also proposed that in smokers the immune system is impaired, with an altered response of macrophages and cytokines, and the inflammatory cascade that occurs in SARS-CoV-2 infection could be especially catastrophic. Another hypothesis is that nicotine may have a protective effect on COVID-19, which disappears due to the abrupt cessation of nicotine intake when smokers are hospitalized. 5,9,10,29-34

Classically, mortality in COPD has been associated with FEV1. It is now known that its predictive value is low when it is greater than $50 \%$ of what was expected and that there are other clinical variables that predict mortality better than FEV1, as reflected in the different multicomponent prognostic indices in COPD. In our work, no association was found between FEV1 and all-cause mortality. ${ }^{35,36}$

Scientific societies advise maintaining the usual treatment and management of exacerbation in COPD patients according to current recommendations. The best way to prevent and reduce the severity of exacerbations in respiratory infections in patients with COPD is optimal drug treatment. There is no evidence that inhaled corticosteroids increase the risk of or worsen SARS-CoV-2 infection. In an exacerbation of COPD with concurrent SARS-CoV-2 infection, it is advisable to follow the indications for treatment with corticosteroids recommended in the guidelines, although efforts should be made to limit the dose and 
duration of the corticosteroids due to the possibility of increasing viral replication. ${ }^{37,38}$

In our work, COPD patients who were in regular treatment with systemic corticosteroids had a higher mortality rate. This could possibly be a reflection of more severe COPD with worse functional capacity in addition to possible immunosuppression associated with the use of systemic corticosteroids, which would entail a higher viral load and greater SARS-CoV-2 involvement.

A better prognosis was not found in patients treated with lopinavir/ritonavir or systemic corticosteroids. The study by Cao et al also found no benefits in the use of lopinavir/ritonavir in hospitalized patients with severe COVID-19 pneumonia, although the study was probably underpowered. $^{39}$

We found a higher survival rate in patients treated with hydroxychloroquine and with macrolides. Azithromycin is frequently used for exacerbations of COPD, but it has emerged as a possible complementary therapy with hydroxychloroquine for COVID-19. Azithromycin has immunomodulatory activity and, presumably, antiviral activity. Hydroxychloroquine would have activity at various levels: on the one hand, it acts through ACE2, attenuating the entry of SARS-CoV-2 into the pulmonary epithelium and, on the other hand, it can have immunosuppressive effects by reducing IL- 6 production in T cells and monocytes. Caution is advised in trials evaluating azithromycin along with hydroxychloroquine for its effects on QT segment prolongation and propensity for arrhythmias. ${ }^{40-42}$ Regarding hydroxychloroquine, our study is not designed to verify its usefulness in treatment and at present, its use is only recommended in clinical trials. Further studies are required to determine the usefulness of azithromycin and/or hydroxychloroquine treatment in COPD patients with SARS-CoV-2 infection.

SARS-COv2 infection produces immunosuppression with lymphopenia, suppression of interferon, and defective NK cell function. This loss of the antiviral defense mechanism can activate a more aggressive "second wave" of immunity, with a cytosine storm, with very high levels of ferritin, C-reactive protein and IL-1 $\beta$, IL-2, IL-6, IL -17, IL-8 and TNF. We also found an elevated D-dimer as a representation of the extension of this hyperinflammatory state to the adjacent microcirculation with secondary fibrinolytic activation that would be associated with extensive pulmonary microthrombosis. This immune hyperactivity is more confined to the lung parenchyma and adjacent bronchial alveolar lymphoid tissue and is associated with the development of an acute respiratory distress syndrome that may require ventilatory support. This condition is similar to the macrophage activation syndrome seen in some systemic diseases, but located in the lung, which is not usually accompanied by organomegaly or disseminated intravascular coagulation. Anti-IL-6 and anti-IL-1 medications are being used to prevent this. In our study, however, so few patients were treated with tocilizumab or anakinra that no conclusions or recommendations can be drawn. ${ }^{43-47}$

With the data we have so far and given the severity of COVID-19, it may be necessary to consider combination treatment for COPD patients with action at two levels: one to avoid the binding of SARS-CoV-2 to lung tissue and another that blocks the cytokine storm that is released. ${ }^{47}$

This work has several limitations. First, the data included in the registry are collected by a large team of researchers. Second, many patients have a history of COPD, but spirometric data are not always included and as such, the registry could include patients who have not been properly diagnosed of COPD. Third, $26 \%$ of non-COPD patients reported being smokers or ex-smokers. Taking into account the underdiagnosis of COPD in the population, it is logical to assume that the prevalence of patients with COPD is higher. Lastly, in the SEMI-COVID registry, data on chronic treatment with inhalers were not included, so we have not been able to analyze if LAMA and/or LABA could have a protective effect on SARS-CoV-2 lung infection. ${ }^{15}$

\section{Conclusion}

Although patients with COPD do not seem to have a higher risk of contracting SARS-CoV-2 infection, they do have a worse prognosis, especially in patients with greater comorbidity. These patients must be identified early in order to establish preventive measures that reduce risk and provide adequate management.

\section{Acknowledgments}

We gratefully acknowledge all the investigators who participate in the SEMI-COVID-19 Registry. A complete list of the SEMI-COVID-19 Network members is provided in the Appendix. We also thank the SEMI-COVID-19 Registry Coordinating Center, S\&H Medical Science Service, for their quality control data, logistic and administrative support.

\section{Disclosure}

The authors declare that they have no conflicts of interest for this work. 


\section{References}

1. Clinical Practice Guideline for the Diagnosis and Treatment of Patients with Chronic Obstructive Pulmonary Disease (COPD) Spanish Guideline for COPD (GesEPOC). Task Force of GesEPOC. Arch Bronconeumol. 2017;53(Supl1):2-64.

2. Almagro P, López F, Cabrera FJ, et al. Soriano JB y Grupos de trabajo de EPOC y Paciente Pluripatológico y Edad Avanzada de la Sociedad Española de Medicina Interna. Comorbidities in patients hospitalized due to chronic obstructive pulmonary disease. A comparative analysis of the ECCO and ESMI studies. Rev Clin Esp. 2012;212(6):281. doi:10.1016/j.rce.2012.02.014

3. Emami A, Javanmardi F, Pirbonyeh N, Akbari A. Prevalence of underlying diseases in hospitalized patients with COVID-19: a systematic review and meta-analysis. Arch Acad Emerg Med. 2020;8(1):e35.

4. Guan WJ, Liang WH, Zhao Y, et al.; China Medical Treatment Expert Group for COVID-19. Comorbidity and its impact on 1590 patients with Covid-19 in China: a nationwide analysis. Eur Respir J. 2020;55(5):2000547. doi:10.1183/13993003.00547-2020.

5. Leung JM, Yang CX, Tam A, et al. ACE-2 expression in the small Airway Epithelia of smokers and COPD patients: implications for COVID-19. Eur Respir J. 2020;55:2000688. doi:10.1183/13993003. 00688-2020

6. Ziegler CGK, Allon SJ, Nyquist SK, et al. SARS-CoV-2 receptor ACE2 is an interferon-stimulated gene in human Airway epithelial cells and is detected in specific cell subsets across tissues. Cell. 2020;181:1016-1035. doi:10.1016/j.cell.2020.04.035

7. Radzikowska U, Ding M, Tan G, et al. Distribution of ACE2, CD147, CD26, and other SARS-CoV-2 associated molecules in tissues and immune cells in health and in asthma, COPD, obesity, hypertension, and COVID-19 risk factors. Allergy. 2020;75(11):2829-2845. doi:10.1111/all.14429

8. Imai Y, Kuba K, Rao S, et al. Angiotensin-converting enzyme 2 protects from severe acute lung failure. Nature. 2005;436:112-116. doi: 10.1038 /nature 03712

9. Zhao Q, Meng M, Kumar R, et al. The impact of COPD and smoking history on the severity of Covid-19: a systemic review and meta-analysis. J Med Virol. 2020. doi:10.1002/jmv.25889

10. Alqahtani JS, Oyelade T, Aldhahir AM, et al. Prevalence, severity and mortality associated with COPD and smoking in patients with COVID-19: a rapid systematic review and meta-analysis. PLoS One. 2020;15(5). doi:10.1371/journal.pone.0233147

11. San Román Terán CM, Guijarro Merino M, Gómez Huelgas R, Montero Ribas L. Hospital Epidemiology of COPD in Spain. Rev Clin Esp. 2007;207(Supl 1):3-7.

12. Casas Rojo JM, Antón Santos JM, Millán Núñez-Cortés J, et al.; for the SEMI-COVID-19 Network. Clinical characteristics of patients hospitalized with COVID-19 in Spain: results from the SEMICOVID-19 network. Rev Clin Esp. 2020. doi:10.1016/j.rce.2020.07.003

13. Xu G, Yang Y, Du Y, et al. Clinical pathway for early diagnosis of COVID-19: updates from experience to evidence-based practice. Clin Rev Allergy Immunol. 2020;24:1-12.

14. Boixeda R, Campins L, Juanola J, Force L. Is chronic obstructive pulmonary disease a protective factor in SARS-CoV-2 infection? The importance of bronchodilator treatment. Rev Clin Esp. 2020;220 (8):526-528. doi:10.1016/j.rce.2020.07.001

15. Yamaya M, Nishimura H, Deng X, et al. Inhibitory effects of glycopyrronium, formoterol, and budesonide on coronavirus HCoV-229E replication and cytokine production by primary cultures of human nasal and tracheal epithelial cells. Respir Investig. 2020;58:155-168. doi:10.1016/j.resinv.2019.12.005

16. Almagro P, Cabrera FJ, Diez-Manglano J, et al.; Working Group on COPD, Spanish Society of Internal Medicine. Comorbidome and short-term prognosis in hospitalised COPD patients: the ESMI study. Eur Respir J. 2015;46:850-853. doi:10.1183/09031936.000 08015 .
17. Roberts CM, Stone RA, Lowe D, Pursey NA, Buckingham RJ. Comorbidities and 90-day outcomes in hospitalized COPD exacerbations. COPD. 2011;8:354-361. doi:10.3109/15412555.2011.600362

18. Alqahtani JS, Njoku CM, Bereznicki B, et al. Risk factors for all-cause hospital readmission following exacerbation of COPD: a systematic review and meta-analysis. Eur Respir Rev. 2020;29:190166. doi:10.1183/16000617.0166-2019

19. Seidu S, Gillies C, Zaccardi F, et al. The impact of obesity on severe disease and mortality in people with SARS-CoV-2: a systematic review and meta-analysis. Endocrinol Diabetes Metab. 2020;14: e00176. doi:10.1002/edm2.176

20. Spelta F, Fratta Pasini AM, Cazzoletti L, Ferrari M. Body weight and mortality in COPD: focus on the obesity paradox. Eat Weight Disord. 2018;23:15-22. doi:10.1007/s40519-017-0456-Z

21. Sokolowska M, Lukasik Z, Agache I, et al. Immunology of COVID-19: mechanisms, clinical outcome, diagnostics and perspectives - a report of the European Academy of Allergy and Clinical Immunology (EAACI). Allergy. 2020;75(10):2445-2476. doi:10.1111/all.14462

22. Ko FW, Ip M, Chan PKS, et al. Viral Etiology of Acute Exacerbations of COPD in Hong Kong. Chest. 2007;132:900-908. doi:10.1378/chest.07-0530

23. Kurai D, Saraya T, Ishii H, Takizawa H. Virus-induced exacerbations in asthma and COPD. Front Microbiol. 2013;4:293.

24. Guan WJ, Ni ZY, Hu Y, et al.; China Medical Treatment Expert Group for Covid-19. Clinical characteristics of coronavirus disease 2019 in China. N Engl J Med. 382;2020:1708-1720. doi:10.1056/ NEJMoa2002032

25. Wang B, Liv R, Lu Z, Huang Y. Does comorbidity increase the risk of patients with COVID-19: evidence from meta-analysis. Aging. 2020;12:6049-6057. doi:10.18632/aging.103000

26. Wang L, He W, Yu X, et al. Coronavirus disease 2019 in elderly patients: characteristics and prognostic factors based on 4-week follow-up. J Infect. 2020;80:639-645. doi:10.1016/j.jinf.2020.03.019

27. Parohan M, Yaghoubi S, Seraji A, Javanbakht MH, Sarraf P, Djalali M. Risk factors for mortality in patients with coronavirus disease 2019 (COVID-19) infection: a systematic review and meta-analysis of observational studies. Aging Male. 2020;8:1-9. doi: $10.1080 / 13685538.2020 .1774748$

28. Lippi G. Chronic obstructive pulmonary disease is associated with severe coronavirus disease 2019 (COVID-19). Respir Med. 2020;167:105941.

29. Cen Y, Chen X, Shen Y, et al. Risk factors for disease progression in mild to moderate COVID-19 patients- a multi-center observational study. Clin Microbiol Infect. 2020;S1198-743X(20)30341-4.

30. Lippi G, Henry BM. Active smoking is not associated with severity of coronavirus disease 2019 (COVID-19). Eur J Intern Med. 2020;75:107-108. doi:10.1016/j.ejim.2020.03.014

31. Guo FR. Active smoking is associated with severity of coronavirus disease 2019 (COVID-19): an update of a meta-analysis. Tob Induc Dis. 2020;18:37. doi:10.18332/tid/121915

32. Lippi G, Sanchis-Gomar F, Henry BM. Active smoking and COVID-19: a double-edged sword. Eur J Intern Med. 2020;S09536205(20)30182-5.

33. Farsalinos K, Barbouni A, Poulas K, Polosa R, Caponnetto P, Niaura R. Current smoking, former smoking, and adverse outcome among hospitalized COVID-19 patients: a systematic review and meta-analysis. Ther Adv Chronic Dis. 2020;11:2040622320935765. doi: $10.1177 / 2040622320935765$

34. Vardavas CI, Nikitara K. COVID-19 and smoking: a systematic review of the evidence. Tob Induc Dis. 2020;18:20. doi:10.18332/ $\mathrm{tid} / 119324$

35. Celli BR. Predictors of Mortality in COPD. Respir Med. 2010;104:773-779. doi:10.1016/j.rmed.2009.12.017

36. Almagro P, Soriano JB, Cabrera FJ, et al.; Working Group on COPD, SpanishSociety of Internal Medicine*. Short and medium term prognosis in patients hospitalized for COPD exacerbation: the CODEX index. Chest. 2014;145(5):972-980. doi:10.1378/chest.13-1328 
37. Attaway A. Management of patients with COPD during the COVID-19 pandemic. Cleve Clin J Med. 2020. doi:10.3949/ccjm.87a.ccc007

38. Bhutani M, Hernandez P, Bourbeau J, et al. KEY HIGHLIGHTS of the Canadian Thoracic Society's position statement on the optimization of chronic obstructive pulmonary disease management during the COVID-19 pandemic. Chest. 2020;S0012-3692(20)31456-2.

39. Cao B, Wang Y, Wen D, et al. A trial of lopinavir-ritonavir in adults hospitalized with severe COVID-19. $N$ Engl $J$ Med. 2020;382:1787-1799. doi:10.1056/NEJMoa2001282

40. Gautret P, Lagier JC, Parola P, et al. Hydroxychloroquine and azithromycin as a treatment of COVID-19: results of an open-label non-randomized clinical trial. Int J Antimicrob Agents. 2020;56 (1):105949. doi:10.1016/j.ijantimicag.2020.105949

41. Devaux CA, Rolain JM, Colson P, Raoult D. New insights on the antiviral effects of chloroquine against coronavirus: what to expect for COVID-19? Int $J$ Antimicrob Agents. 2020;55:105938. doi:10.1016/j.ijantimicag.2020.105938

42. Tripathy S, Dassarma B, Roy S, Chabalala H, Matsabisa MG. A review on possible modes of action of chloroquine/hydroxychloroquine: repurposing against SAR-CoV-2 (COVID-19) pandemic. Int $J$ Antimicrob Agents. 2020;56:106028. doi:10.1016/j.ijantimicag. 2020.106028
43. McGonagle D, Sharif K, O'Regan A, et al. The role of cytokines including interleukin-6 in COVID-19 induced pneumonia and macrophage activation syndrome-like disease. Autoimmun Rev. 2020;19:102537. doi:10.1016/j.autrev.2020.102537

44. Xu X, Han M, Li T, et al. Effective treatment of severe COVID-19 patients with tocilizumab. Proc Natl Acad Sci U S A. 2020;117 (20):10970-10975. doi:10.1073/pnas.2005615117

45. Toniati P, Piva S, Cattalini M, et al. Tocilizumab for the treatment of severe COVID-19 pneumonia with hyperinflammatory syndrome and acute respiratory failure: a single center study of 100 patients in Brescia, Italy. Autoimmun Rev. 2020;19(7):102568. doi:10.1016/j.autrev.2020. 102568

46. Cavalli G, De Luca G, Campochiaro C, et al. Interleukin-1 blockade with high-dose anakinra in patients with COVID-19, acute respiratory distress syndrome, and hyperinflammation: a retrospective cohort study. Lancet Rheumatol. 2020;2:e325-e331. doi:10.1016/ S2665-9913(20)30127-2

47. Lipworth B, Chan R, Lipworth S, Rui C, Kuo W. Weathering the cytokine storm in susceptible patients with severe SARS-CoV-2 infection. J Allergy Clin Immunol Pract. 2020;8(6):1798-1801. doi:10.1016/j.jaip.2020.04.014

\section{Publish your work in this journal}

The International Journal of COPD is an international, peer-reviewed journal of therapeutics and pharmacology focusing on concise rapid reporting of clinical studies and reviews in COPD. Special focus is given to the pathophysiological processes underlying the disease, intervention programs, patient focused education, and self management protocols. This journal is indexed on PubMed Central, MedLine and CAS. The manuscript management system is completely online and includes a very quick and fair peer-review system, which is all easy to use. Visit http://www.dovepress.com/testimonials.php to read real quotes from published authors. 